\title{
ON LOCAL PROPERTIES OF COMPACTLY SUPPORTED SOLUTIONS OF THE TWO-COEFFICIENT DILATION EQUATION
}

\author{
JANUSZ MORAWIEC
}

Received 22 October 2001

\begin{abstract}
Let $a$ and $b$ be reals. We consider the compactly supported solutions $\varphi: \mathbb{R} \rightarrow \mathbb{R}$ of the twocoefficient dilation equation $\varphi(x)=a \varphi(2 x)+b \varphi(2 x-1)$. In this paper, we determine sets $B_{a, b}, C_{a, b}$, and $Z_{a, b}$ defined in the following way: let $x \in[0,1]$. We say that $x \in B_{a, b}$ (resp., $x \in C_{a, b}, x \in Z_{a, b}$ ) if the zero function is the only compactly supported solution of the two-coefficient dilation equation, which is bounded in a neighbourhood of $x$ (resp., continuous at $x$, vanishes in a neighbourhood of $x$ ). We also give the structure of the general compactly supported solution of the two-coefficient dilation equation.
\end{abstract}

2000 Mathematics Subject Classification: 39B12, 39B22.

1. Introduction. The two-coefficient dilation equation is a functional equation of the form

$$
\varphi(x)=a \varphi(2 x)+b \varphi(2 x-1) .
$$

This equation is the simplest case of the so-called dilation equation

$$
\varphi(x)=\sum_{n=0}^{N} c_{n} \varphi(2 x-n),
$$

where $N$ is a positive integer and $c_{0}, \ldots, c_{N}$ are real (or complex) constants. Equation (1.2) is also referred to as the two-scale difference equation or the refinement equation. A nonzero solution of (1.2) is called a scaling function. For a deeper discussion of (1.2), and some related references, we refer the reader to Benedetto and Frazier [2, Chapter 4].

It is well known that the characteristic function of the interval $[0,1)$ is a scaling function related to (1.1) with $a=b=1$. This scaling function generates the simplest known wavelet called the Haar wavelet (see, e.g., [3] or [4]). Haar [7] found this wavelet long before the word wavelet has been introduced.

It is also known that if $\varphi: \mathbb{R} \rightarrow \mathbb{R}$ is a nontrivial and compactly supported $L^{1}$-solution of (1.1), then $a=b=1$ and there exists a real constant $c \neq 0$ such that $\varphi=c \chi_{[0,1)}$ almost everywhere (see [5]). Recently, Pittenger and Ryff [9] proved that the above result is still true if we assume that $\varphi$ is measurable instead of $L^{1}$.

On the other hand, for every nonzero reals $a$ and $b,(1.1)$ passes very irregular scaling functions. More precisely, if $a b \neq 0$, then (1.1) has compactly supported solution such that its graph meets every Borel subset of $[0,1] \times \mathbb{R}$ with uncountable vertical projection (see [8]). Each such function (called a function with a big graph) has rather 
strange properties. In particular, if $\varphi_{0}:[0,1] \rightarrow \mathbb{R}$ is a function with a big graph, then the graph of $\varphi_{0}$ is connected and the set $([0,1] \times \mathbb{R}) \backslash \operatorname{graph}\left(\varphi_{0}\right)$ contains no subset of $[0,1] \times \mathbb{R}$ of second category having the property of Baire, and contains no subset of $[0,1] \times \mathbb{R}$ of positive inner Lebesgue measure (see [1]).

It is also proved in [8] that if $a b \neq 0$ and if $|a|>1$ or $|b|>1$, then every compactly supported scaling function of (1.1) is rather irregular in the sense that it is unbounded in every neighbourhood of each point of $[0,1]$.

The purpose of this paper is to determine all reals $a$ and $b$ for which every compactly supported scaling function of (1.1) is irregular in the above sense.

2. Notation. We make the following definition.

Definition 2.1. For $a, b \in \mathbb{R}$ by $B_{a, b}$ denote the set of all $x \in[0,1]$ such that the zero function is the only compactly supported solution of (1.1) which is bounded in a neighbourhood of $x$.

By $C_{a, b}$ denote the set of all $x \in[0,1]$ such that the zero function is the only compactly supported solution of (1.1) which is continuous at $x$.

And by $Z_{a, b}$ denote the set of all $x \in[0,1]$ such that the zero function is the only compactly supported solution of (1.1) which vanishes in a neighbourhood of $x$.

In the definition, we restrict ourselves to points from $[0,1]$ only, because of assertion (i) of Lemma 2.2 which we repeat from [8] without proof.

LEMmA 2.2. Assume that $a$ and $b$ are reals and let $\varphi: \mathbb{R} \rightarrow \mathbb{R}$ be a compactly supported solution of (1.1). Then

(i) $\operatorname{supp} \varphi \subset[0,1]$;

(ii) for every $x \in(0,1)$, every positive integer $n$ and any $\varepsilon_{1}, \ldots, \varepsilon_{n} \in\{0,1\}$,

$$
\varphi\left(\frac{x}{2^{n}}+\sum_{i=1}^{n} \frac{\varepsilon_{i}}{2^{i}}\right)=a^{n-N} b^{N} \varphi(x),
$$

where $N=\operatorname{card}\left\{i \in\{1, \ldots, n\} \mid \varepsilon_{i}=1\right\}$;

(iii) if $b=0$, then $\left.\varphi\right|_{\mathbb{R} \backslash\{0\}}=0$. If $a=0$, then $\left.\varphi\right|_{\mathbb{R} \backslash\{1\}}=0$;

(iv) the function $\psi: \mathbb{R} \rightarrow \mathbb{R}$ defined by $\psi(x)=\varphi(1-x)$ is compactly supported and satisfies

$$
\psi(x)=b \psi(2 x)+a \psi(2 x-1)
$$

for every $x \in \mathbb{R}$.

It is clear that

$$
B_{a, b} \subset C_{a, b} \subset Z_{a, b} \subset[0,1],
$$

for any reals $a$ and $b$. Moreover, from assertion (iv) of Lemma 2.2, we conclude that

$$
B_{a, b}=1-B_{b, a}, \quad C_{a, b}=1-C_{b, a}, \quad Z_{a, b}=1-Z_{b, a},
$$

for any reals $a$ and $b$. 
3. General compactly supported solution. We need only to consider the case where $a b \neq 0$, because of assertion (iii) of Lemma 2.2.

We begin with some elementary properties of compactly supported solutions of (1.1).

LEMmA 3.1. Assume that $a b \neq 0$. Let $x, y \in(0,1)$ and let $\varphi: \mathbb{R} \rightarrow \mathbb{R}$ be a compactly supported solution of (1.1). Then

(i) if $x=z / 2^{k}+\sum_{i=1}^{k} \alpha_{i} / 2^{i}$ and $y=z / 2^{l}+\sum_{i=1}^{l} \beta_{i} / 2^{i}$ with some $z \in(0,1)$, positive integers $k, l$ and $\alpha_{1}, \ldots, \alpha_{k}, \beta_{1}, \ldots, \beta_{l} \in\{0,1\}$, then

$$
\varphi(y)=a^{l-k+K-L} b^{L-K} \varphi(x)
$$

where

$$
\begin{gathered}
K=\operatorname{card}\left\{i \in\{1, \ldots, k\} \mid \alpha_{i}=1\right\}, \\
L=\operatorname{card}\left\{i \in\{1, \ldots, l\} \mid \beta_{i}=1\right\} ;
\end{gathered}
$$

(ii) if $y=y / 2^{l}+\sum_{i=1}^{l} \beta_{i} / 2^{i}$ with some positive integer $l$ and $\beta_{1}, \ldots, \beta_{l} \in\{0,1\}$ and if $a^{l-L} b^{L} \neq 1$ with $L$ defined by (3.3), then $\varphi(y)=0$;

(iii) for every nonnegative integer $l$ and any $\beta_{1}, \ldots, \beta_{l} \in\{0,1\}$,

$$
\varphi\left(\frac{1}{2^{l+1}}+\sum_{i=1}^{l} \frac{\beta_{i}}{2^{i}}\right)=a^{l-L} b^{L}[a \varphi(1)+b \varphi(0)],
$$

with $L$ defined by (3.3);

(iv) if $a \neq 1$, then $\varphi(0)=0$. If $b \neq 1$, then $\varphi(1)=0$.

Proof. To prove (i), observe that from assertion (ii) of Lemma 2.2 we have

$$
\varphi(x)=a^{k-K} b^{K} \varphi(z)
$$

with $K$ defined by (3.2) and

$$
\varphi(y)=a^{l-L} b^{L} \varphi(z)
$$

with $L$ defined by (3.3). Since $a b \neq 0$, we obtain (3.1), by combining (3.5) with (3.6).

Replacing $z$ by $y$ in (3.6) and assuming that $a^{l-L} b^{L} \neq 1$, we conclude that $\varphi(y)=0$ which proves (ii).

The proof of (iii) is by induction on $l$.

To see that (3.4) holds for $l=0$, it is enough to put $x=1 / 2$ in (1.1).

Fix a nonnegative integer $l$ and suppose that (3.4) is satisfied for any $\beta_{1}, \ldots, \beta_{l} \in$ $\{0,1\}$ with $L$ defined by (3.3). We will show that

$$
\varphi\left(\frac{1}{2^{l+2}}+\sum_{i=1}^{l+1} \frac{\varepsilon_{i}}{2^{i}}\right)=a^{l+1-L_{1}} b^{L_{1}}[a \varphi(1)+b \varphi(0)],
$$


for any $\varepsilon_{1}, \ldots, \varepsilon_{l+1} \in\{0,1\}$, where

$$
L_{1}=\operatorname{card}\left\{i \in\{1, \ldots, l+1\} \mid \varepsilon_{i}=1\right\}
$$

Fix $\varepsilon_{1}, \ldots, \varepsilon_{l+1} \in\{0,1\}$ and put

$$
\beta_{i}=\varepsilon_{i+1}, \quad \forall i \in\{1, \ldots, l\}
$$

If $\varepsilon_{1}=0$, then $1 / 2^{l+2}+\sum_{i=1}^{l+1} \varepsilon_{i} / 2^{i} \in(0,1 / 2)$, whence $2\left(1 / 2^{l+2}+\sum_{i=1}^{l+1} \varepsilon_{i} / 2^{i}\right)-1<0$. Moreover, $2\left(1 / 2^{l+2}+\sum_{i=1}^{l+1} \varepsilon_{i} / 2^{i}\right)=1 / 2^{l+1}+\sum_{i=1}^{l} \beta_{i} / 2^{i}$. Hence, by assertion (i) of Lemma 2.2 and (3.4), we get

$$
\varphi\left(\frac{1}{2^{l+2}}+\sum_{i=1}^{l+1} \frac{\varepsilon_{i}}{2^{i}}\right)=a \varphi\left(\frac{1}{2^{l+1}}+\sum_{i=1}^{l} \frac{\beta_{i}}{2^{i}}\right)=a^{l+1-L} b^{L}[a \varphi(1)+b \varphi(0)] .
$$

To conclude that (3.7) holds it is enough to observe that, by (3.3), (3.8), (3.9), and the fact that $\varepsilon_{1}=0$, we have $L=L_{1}$.

Similarly, if $\varepsilon_{1}=1$, then $1 / 2^{l+2}+\sum_{i=1}^{l+1} \varepsilon_{i} / 2^{i} \in(1 / 2,1)$, whence $2\left(1 / 2^{l+2}+\right.$ $\left.\sum_{i=1}^{l+1} \varepsilon_{i} / 2^{i}\right)>1$. Moreover $2\left(1 / 2^{l+2}+\sum_{i=1}^{l+1} \varepsilon_{i} / 2^{i}\right)-1=1 / 2^{l+1}+\sum_{i=1}^{l} \beta_{i} / 2^{i}$. Hence, by assertion (i) of Lemma 2.2 and (3.4), we get

$$
\varphi\left(\frac{1}{2^{l+2}}+\sum_{i=1}^{l+1} \frac{\varepsilon_{i}}{2^{i}}\right)=b \varphi\left(\frac{1}{2^{l+1}}+\sum_{i=1}^{l} \frac{\beta_{i}}{2^{i}}\right)=a^{l+1-(L+1)} b^{L+1}[a \varphi(1)+b \varphi(0)]
$$

To conclude that (3.7) holds also in this case, it is enough to observe that, by (3.3), (3.8), (3.9), and the fact that $\varepsilon_{1}=1$, we now have $L+1=L_{1}$.

To get (iv) notice that, by assertion (i) of Lemma 2.2, we have $\varphi(0)=a \varphi(0)$ and $\varphi(1)=b \varphi(1)$. Therefore if $a \neq 1$, then $\varphi(0)=0$, and if $b \neq 1$, then $\varphi(1)=0$.

From now on, let

$$
\mathbf{M}=\left\{\frac{p}{2^{k}} \mid p, k \in \mathbb{Z}\right\}
$$

and let $\sim$ be an equivalence relation on $\mathbb{R}$ defined by

$$
x \sim y \Longleftrightarrow \text { there exists an integer } k \text { such that } 2^{k} x-y \in \mathbf{M} \text {. }
$$

Let $[x]$ denote the equivalence class of $x$. This equivalence relation has previously been used by Förg-Rob [6]. The next lemma can be found in [6]. 
LEMMA 3.2. If $x \in(0,1) \backslash \mathbf{M}$ and $y \in(0,1)$, then $x \sim y$ if and only if there are some $z \in(0,1)$, positive integers $k, l$, and nonnegative integers $m, n$ such that $m<2^{k}$, $n<2^{l}, x=(z+m) / 2^{k}$ and $y=(z+n) / 2^{l}$.

The general compactly supported solution of (1.1) can be obtained by describing it on every equivalence class of the relation $\sim$. The next two theorems show how to do it.

THEOREM 3.3. Assume that $a b \neq 0$. The general compactly supported solution of (1.1) on the set $\mathbf{M}$ can be obtained in the following way. Let $\left.\varphi\right|_{\mathbf{M} \backslash[0,1]}=0$ and

(i) if $a \neq 1$ and $b \neq 1$, then $\left.\varphi\right|_{\mathrm{M} \cap[0,1]}=0$;

(ii) if $a \neq 1$ and $b=1$, then $\varphi(0)=0$, choose arbitrarily $\varphi(1)$ and for every nonnegative integer $l$ and for any $\beta_{1}, \ldots, \beta_{l} \in\{0,1\}$ accept (3.4) with $L$ defined by (3.3);

(iii) if $a=1$ and $b \neq 1$, then $\varphi(1)=0$, choose arbitrarily $\varphi(0)$ and for every nonnegative integer $l$ and for any $\beta_{1}, \ldots, \beta_{l} \in\{0,1\}$ accept (3.4) with $L$ defined by (3.3);

(iv) if $a=1$ and $b=1$, then choose arbitrarily $\varphi(0)$ and $\varphi(1)$, and for every nonnegative integer $l$ and for any $\beta_{1}, \ldots, \beta_{l} \in\{0,1\}$ accept (3.4) with $L$ defined by (3.3).

Proof. According to assertions (iii) and (iv) of Lemma 3.1, it is enough to show that the function $\varphi$, defined in each of the cases (i), (ii), (iii), and (iv), satisfies (1.1) for every $x \in \mathbf{M}$. The proof of this fact is similar to the proof of assertion (iii) of Lemma 3.1, so we omit it.

THEOREM 3.4. Assume that $a b \neq 0$ and let $x \in(0,1) \backslash \mathbf{M}$. The general compactly supported solution of (1.1) on $[x]$ can be obtained in the following way. Let $\left.\varphi\right|_{[x] \backslash(0,1)}=0$ and consider two cases:

(i) there exists some $y \in[x] \cap(0,1)$ such that $y=y / 2^{l}+\sum_{i=1}^{l} \beta_{i} / 2^{i}$, where $l$ is a positive integer and $\beta_{1}, \ldots, \beta_{l} \in\{0,1\}$, and $a^{l-L} b^{L} \neq 1$ with $L$ defined by (3.3). Then, let $\left.\varphi\right|_{[x] \cap(0,1)}=0$;

(ii) the first case does not hold. Choose arbitrarily $\varphi(x)$, represent $x$ as $x=z / 2^{k}+$ $\sum_{i=1}^{k} \alpha_{i} / 2^{i}$ with some $z \in(0,1)$, a positive integer $k$ and $\alpha_{1}, \ldots, \alpha_{k} \in\{0,1\}$, and for every $y=z / 2^{l}+\sum_{i=1}^{l} \beta_{i} / 2^{i}$, where $l$ is a positive integer and $\beta_{1}, \ldots, \beta_{l} \in$ $\{0,1\}$, put (3.1) with $K$ defined by (3.2) and $L$ defined by (3.3).

Proof. On account of assertions (i) and (ii) of Lemma 3.1, it is sufficient to prove that the function $\varphi$ given by (3.1) is well defined and $\varphi(y)=a \varphi(2 y)+b \varphi(2 y-1)$ for every $y \in[x]$. The proofs of these two facts can be adapted from the proof of $[8$, Lemma 3].

4. Local properties of compactly supported solutions. As a consequence of assertion (iii) of Lemma 2.2 (see also (2.4)) we obtain the following result concerning the case where $a b=0$.

REMARK 4.1. Observe that

$$
\begin{gathered}
B_{1,0}=B_{0,1}=\varnothing . \\
C_{1,0}=Z_{1,0}=\{0\}, \quad C_{0,1}=Z_{0,1}=\{1\} .
\end{gathered}
$$


and if $a \neq 1$, then

$$
B_{a, 0}=C_{a, 0}=Z_{a, 0}=B_{0, a}=C_{0, a}=Z_{0, a}=[0,1] .
$$

To determine the sets $B_{a, b}, C_{a, b}$, and $Z_{a, b}$ in the case where $a b \neq 0$ we will need four lemmas. The first one can be found in [8, Theorem 1].

LEMMA 4.2. Assume that $a b \neq 0$. If $|a|>1$ or $|b|>1$, then every compactly supported solution of (1.1), which is bounded in a neighbourhood of a point of [0,1], vanishes everywhere.

Proofs of the next three lemmas are similar to each other.

LEMmA 4.3. Assume that $a b \neq 0$. If $|a|<1$ and $|b|<1$, then every compactly supported solution of (1.1), which is bounded in a neighbourhood of a point of [0,1], vanishes everywhere.

Proof. On account of assertion (iv) of Lemma 2.2, it is sufficient to consider only the case $|a / b| \geq 1$.

Let $\varphi: \mathbb{R} \rightarrow \mathbb{R}$ be a compactly supported solution of (1.1) which is bounded in a neighbourhood $U$ of a point $x_{0} \in[0,1]$. Since $\left\{m / 2^{n} \mid n \in \mathbb{N}, m \in\left\{0, \ldots, 2^{n}-1\right\}\right\}$ is a dense subset of $[0,1]$, we may (and do) assume that there are a positive integer $l$ and $\beta_{1}, \ldots, \beta_{l} \in\{0,1\}$ such that

$$
x_{0}=\sum_{i=1}^{l} \frac{\beta_{i}}{2^{i}} \in(0,1), \quad \frac{z}{2^{l}}+x_{0} \in U \cap(0,1), \quad \forall z \in(0,1) .
$$

Fix $x \in[0,1]$. According to assertion (i) of Lemma 2.2, the proof will be finished if we show that $\varphi(x)=0$.

If $x \in \mathbf{M}$, then $\varphi(x)=0$, by assertions (iv) and (iii) of Lemma 3.1 (in this case we do not need the boundedness of $\varphi$ on $U$ ).

Now let $x \in(0,1) \backslash \mathbf{M}$. Then for every positive integer $n$, there are some $z_{n} \in(0,1)$ and $\alpha_{n, 1}, \ldots, \alpha_{n, l+n} \in\{0,1\}$ such that

$$
x=\frac{z_{n}}{2^{l+n}}+\sum_{i=1}^{l+n} \frac{\alpha_{n, i}}{2^{i}} .
$$

Applying assertion (i) of Lemma 3.1 (with $y=z_{n} / 2^{l}+x_{0}$ ), we have

$$
\varphi\left(\frac{z_{n}}{2^{l}}+x_{0}\right)=a^{l-(l+n)+L_{n}-L} b^{L-L_{n}} \varphi(x)=\left(\frac{1}{a}\right)^{n}\left(\frac{a}{b}\right)^{L_{n}-L} \varphi(x),
$$

with $L$ given by (3.3) and $L_{n}=\operatorname{card}\left\{i \in\{1, \ldots, l+n\} \mid \alpha_{n, i}=1\right\}$. Since the left-hand side of (4.5) is bounded with respect to $n$, we conclude that $\varphi(x)=0$, and the proof is complete.

LEMmA 4.4. Assume that $a b \neq 0$. If $|b|<|a|=1$ or $|a|<|b|=1$, then every compactly supported solution of (1.1), which is bounded in a neighbourhood of a point of $[0,1]$, vanishes outside of the set $\mathbf{M} \cap[0,1]$. 
Proof. According to assertion (iv) of Lemma 2.2, we can assume that

$$
|a|=1, \quad|b|<1 .
$$

Let $\varphi: \mathbb{R} \rightarrow \mathbb{R}$ be a compactly supported solution of (1.1), which is bounded in a neighbourhood $U$ of a point of $[0,1]$. Without loss of restriction, we can assume that there are a positive integer $k$ and $\varepsilon_{1}, \ldots, \varepsilon_{k} \in\{0,1\}$ such that

$$
U=\left(\sum_{i=1}^{k} \frac{\varepsilon_{i}}{2^{i}}, \sum_{i=1}^{k} \frac{\varepsilon_{i}}{2^{i}}+\frac{1}{2^{k}}\right) .
$$

Fix $x \in(0,1) \backslash \mathbf{M}$. Since the set $[x]$ is a dense subset of $\mathbb{R}$ we choose a $y \in[x] \cap U$. On account of assertion (i) of Lemma 2.2, Lemma 3.2, and assertion (i) of Lemma 3.1 it is enough to show that $\varphi(y)=0$.

Clearly, for every positive integer $n$ there are some $z_{n} \in(0,1)$ and $\alpha_{n, k+1}, \ldots, \alpha_{n, k+n}$ $\in\{0,1\}$ such that

$$
y=\frac{z_{n}}{2^{k+n}}+\sum_{i=1}^{k} \frac{\varepsilon_{i}}{2^{i}}+\sum_{i=k+1}^{k+n} \frac{\alpha_{n, i}}{2^{i}} .
$$

Let $L_{n}=\operatorname{card}\left\{i \in\{k+1, \ldots, k+n\} \mid \alpha_{n, i}=1\right\}$ for every positive integer $n$. The sequence $\left(L_{n}: n \in \mathbb{N}\right)$ is increasing and

$$
\lim _{n \rightarrow \infty} L_{n}=+\infty
$$

Now, for every positive integer $n$, we put

$$
y_{n}=\frac{z_{n}}{2^{k+n}}+\sum_{i=1}^{k} \frac{\varepsilon_{i}}{2^{i}},
$$

and observe that $y_{n} \in U$. Moreover, by assertion (i) of Lemma 3.1, we have

$$
\varphi\left(y_{n}\right)=\left(\frac{a}{b}\right)^{L_{n}} \varphi(y)
$$

Since the left-hand side of (4.11) is bounded with respect to $n$, we conclude from (4.6) and (4.9) that $\varphi(y)=0$, which completes the proof.

LEMMA 4.5. Let $(a, b) \in\{(-1,1),(1,-1),(-1,-1)\}$, let $\varphi$ be a compactly supported solution of (1.1), and let $x \in(0,1)$. Let

$$
\begin{aligned}
& S_{+}=\{y \in[x] \cap(0,1): \varphi(y)=\varphi(x)\}, \\
& S_{-}=\{y \in[x] \cap(0,1): \varphi(y)=-\varphi(x)\} .
\end{aligned}
$$

Then, $S_{+} \cup S_{-}=[x] \cap(0,1)$ and $S_{+}$and $S_{-}$are dense subsets of $[0,1]$. 
Proof. By Lemmas 3.1 and 3.2, it is evident that $S_{+} \cup S_{-}=[x] \cap(0,1)$. It remains to prove that $S_{+}$and $S_{-}$are dense subsets of $[0,1]$. Since $[x] \cap(0,1)$ is a dense subset of $[0,1]$, it is sufficient to show that for every $y \in[x] \cap(0,1)$ and for every positive integer $n$, there exists some $y_{n} \in[x] \cap(0,1)$ such that $\left|y-y_{n}\right|<1 / 2^{n}$ and $\varphi\left(y_{n}\right)=$ $-\varphi(y)$.

We first assume that $x \in \mathbf{M} \cap(0,1)$. Fix $y \in \mathbf{M} \cap(0,1)$ and write it in the form

$$
y=\frac{1}{2^{l+1}}+\sum_{i=1}^{l} \frac{\beta_{i}}{2^{i}}
$$

where $l$ is a nonnegative integer and $\beta_{1}, \ldots, \beta_{l} \in\{0,1\}$. For every positive integer $n \geq 2$, we put

$$
y_{n}=\frac{1}{2^{l+n+1}}+\sum_{i=1}^{l} \frac{\beta_{i}}{2^{i}}+\sum_{i=l+2}^{l+n} \frac{1}{2^{i}},
$$

and observe that $y_{n} \in \mathbf{M} \cap(0,1)$ and $\left|y-y_{n}\right|=1 / 2^{l+n+1}<1 / 2^{n}$. Moreover, assertion (iii) of Lemma 3.1 gives

$$
\varphi\left(y_{n}\right)=a b^{n-1} \varphi(y)
$$

Thus $\varphi\left(y_{2 n+1}\right)=-\varphi(y)$, if $a=-1$ and $\varphi\left(y_{2 n}\right)=-\varphi(y)$, if $a=1$.

Now, we assume that $x \in(0,1) \backslash$ M. Fix $y \in[x] \cap(0,1)$ and assume that

$$
y=\frac{z}{2^{k}}+\sum_{i=1}^{k} \frac{\varepsilon_{i}}{2^{i}},
$$

where $k$ is a positive integer, $z \in(0,1)$ and $\varepsilon_{1}, \ldots, \varepsilon_{k} \in\{0,1\}$. Clearly, for every positive integer $n$ there are some $z_{n} \in(0,1)$ and $\alpha_{n, k+1}, \ldots, \alpha_{n, k+n} \in\{0,1\}$ such that (4.8) holds. For every positive integers $n$, we put

$$
y_{n}=\frac{z_{n}}{2^{k+4 n}}+\sum_{i=1}^{k} \frac{\varepsilon_{i}}{2^{i}}+\sum_{i=k+1}^{k+n} \frac{\alpha_{n, i}}{2^{i}}+\frac{1}{2^{k+2 n}}
$$

and observe that $\left|y-y_{n}\right|<1 / 2^{n}$ and $y_{n} \in[x] \cap(0,1)$, by Lemma 3.2. Moreover, assertion (i) of Lemma 3.1 gives

$$
\varphi\left(y_{n}\right)=a^{3 n-1} b \varphi(y)
$$

Thus $\varphi\left(y_{2 n+1}\right)=-\varphi(y)$, if $b=-1$ and $\varphi\left(y_{2 n}\right)=-\varphi(y)$, if $b=1$.

The proof is complete.

Now, we can formulate our main result. 
THEOREM 4.6. Assume that $a b \neq 0$.

(i) If $|a| \leq b=1$ or $|b| \leq a=1$ or $a=b=-1$, then $B_{a, b}=\varnothing$.

(ii) If $\max \{|a|,|b|\} \neq 1$ or $|a|<1=-b$ or $|b|<1=-a$, then $B_{a, b}=[0,1]$.

(iii) The sets $C_{1,1}$ and $Z_{1,1}$ consist of two elements only, 0 and 1 .

(iv) If $a \neq 1$ or $b \neq 1$, then $C_{a, b}=Z_{a, b}=[0,1]$.

Proof. Assertion (i) follows from Theorems 3.3 and 3.4, which allow (in each of the considered cases) to construct a nonzero, bounded and compactly supported solution of (1.1).

To get assertion (ii), it is sufficient to use Lemmas 4.2, 4.3, and 4.4 with assertions (iv) and (iii) of Lemma 3.1.

Now, let $a=b=1$. First, observe that since the function $\varphi: \mathbb{R} \rightarrow \mathbb{R}$, given by $\varphi(0)=1, \varphi(1)=-1$ and $\varphi(x)=0$ for every $x \in \mathbb{R} \backslash\{0,1\}$, is a compactly supported solution of (1.1), we have $Z_{1,1} \subset\{0,1\}$. Hence, according to (2.3) and (2.4), the proof of (iii) will be completed if we show that $0 \in C_{1,1}$. For this purpose, assume that $\varphi$ is a compactly supported solution of (1.1) which is continuous at 0 . This jointly with assertion (i) of Lemma 2.2 implies that $\varphi$ vanishes outside of $(0,1]$. Moreover, from Lemmas 3.2 and 3.1, we see that for every $x \in[0,1]$ the function $\left.\varphi\right|_{[x] \cap(0,1)}$ is constant and since $[x] \cap(0,1)$ is a dense subset of $[0,1]$, we conclude that $\left.\varphi\right|_{[x] \cap(0,1)}=0$. Consequently, $\varphi$ vanishes everywhere, except the point 1 . To get that $\varphi(1)=0$, it is enough to use assertion (iii) of Lemma 3.1.

Using similar argumentation as above, we conclude that assertion (iv) follows from Lemma 4.5 in the case $(a, b) \in\{(-1,1),(1,-1),(-1,-1)\}$; otherwise it is enough to use Lemmas 4.2, 4.3, and 4.4 with assertion (iii) of Lemma 3.1.

We finish with two propositions which proofs are left to the reader.

Proposition 4.7. For every reals $a$ and $b$, every compactly supported solution of (1.1) is either bounded on $\mathbb{R}$ or unbounded in every neighbourhood of each point of $[0,1]$.

Proposition 4.8. For every reals $a$ and $b$, every compactly supported solution of (1.1) is either constant on $(0,1)$ or discontinuous at every point of $[0,1]$.

AcKNowledgment. This research was supported by the State Committee for Scientific Research Grant No. 2 P03A 03311.

\section{REFERENCES}

[1] L. Bartłomiejczyk, Solutions with big graph of homogeneous functional equations in a single variable, Aequationes Math. 56 (1998), no. 1-2, 149-156.

[2] J. J. Benedetto and M. W. Frazier (eds.), Wavelets: Mathematics and Applications, Studies in Advanced Mathematics, CRC Press, Florida, 1994.

[3] C. K. Chui, An Introduction to Wavelets, Wavelet Analysis and Its Applications, vol. 1, Academic Press, Massachusetts, 1992.

[4] I. Daubechies, Ten Lectures on Wavelets, CBMS-NSF Regional Conference Series in Applied Mathematics, vol. 61, Society for Industrial and Applied Mathematics (SIAM), Pennsylvania, 1992.

[5] I. Daubechies and J. C. Lagarias, Two-scale difference equations. I. Existence and global regularity of solutions, SIAM J. Math. Anal. 22 (1991), no. 5, 1388-1410. 
[6] W. Förg-Rob, On a problem of R. Schilling. II, Math. Pannon. 5 (1994), no. 2, 145-168.

[7] A. Haar, Zur Theorie der orthogonalen Funktionensysteme, Math. Ann. 69 (1910), 331-371 (German).

[8] J. Morawiec, On the existence of irregular solutions of the two-coefficient dilation equation, Aequationes Math. 62 (2001), no. 1-2, 79-84.

[9] A. O. Pittenger and J. V. Ryff, A probabilistic approach to scaling equations, Aequationes Math. 58 (1999), no. 3, 242-259.

JANUSZ MoRAWIEC: INSTYTUT MATEMATYKI, UNIWERSYTET ŚLA̧SKI, UL. BANKOWA 14, PL-40007 Katowice, POLAND

E-mail address: morawiec@ux2.math.us.edu.p1 


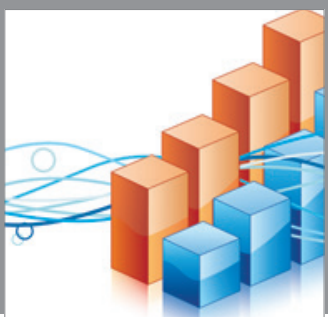

Advances in

Operations Research

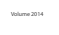

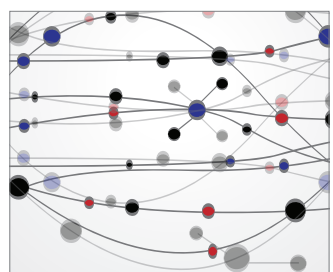

\section{The Scientific} World Journal
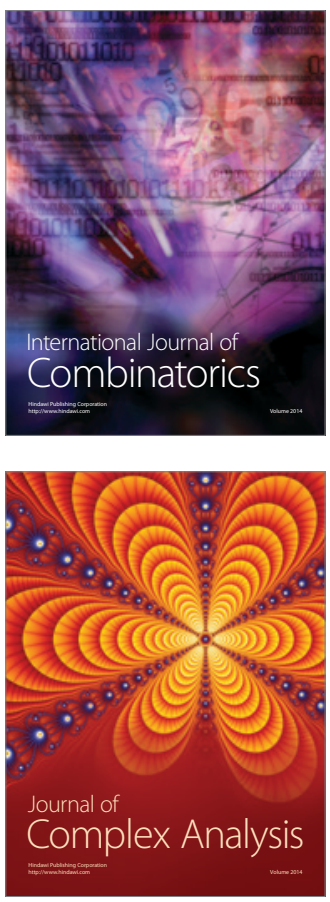

International Journal of

Mathematics and

Mathematical

Sciences
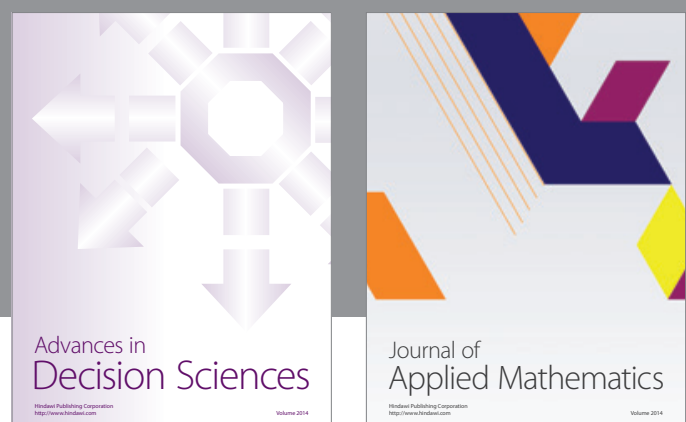

Journal of

Applied Mathematics
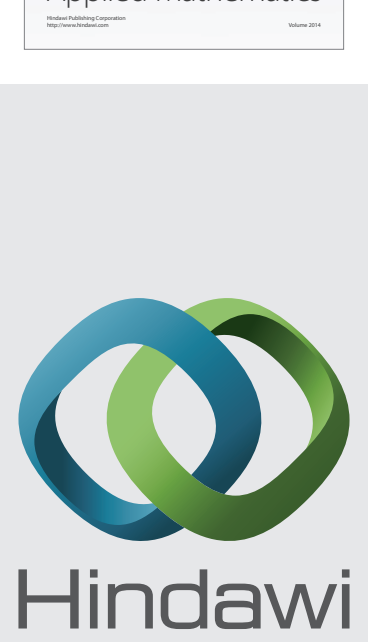

Submit your manuscripts at http://www.hindawi.com
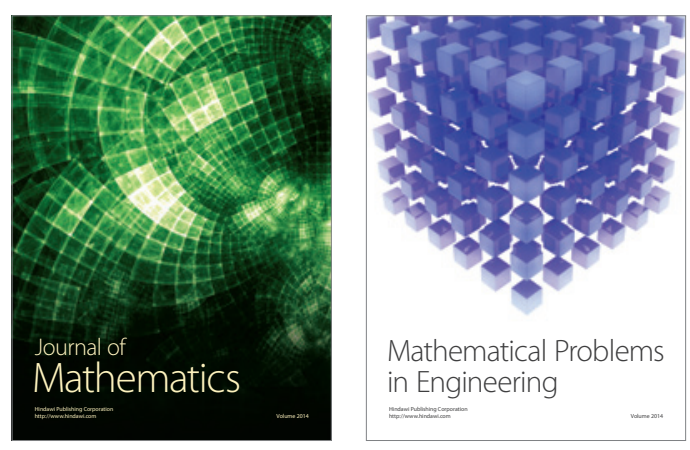

Mathematical Problems in Engineering
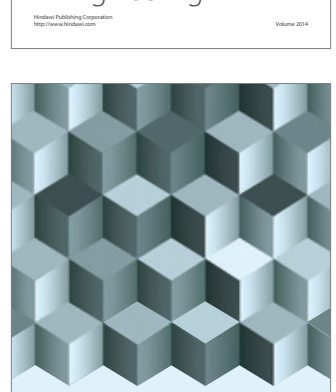

Journal of

Function Spaces
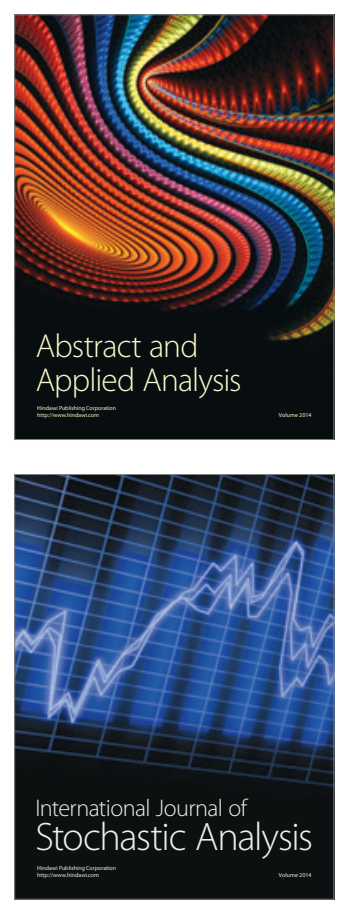

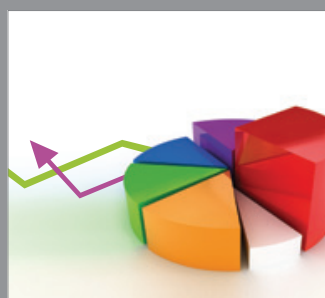

ournal of

Probability and Statistics

Promensencen
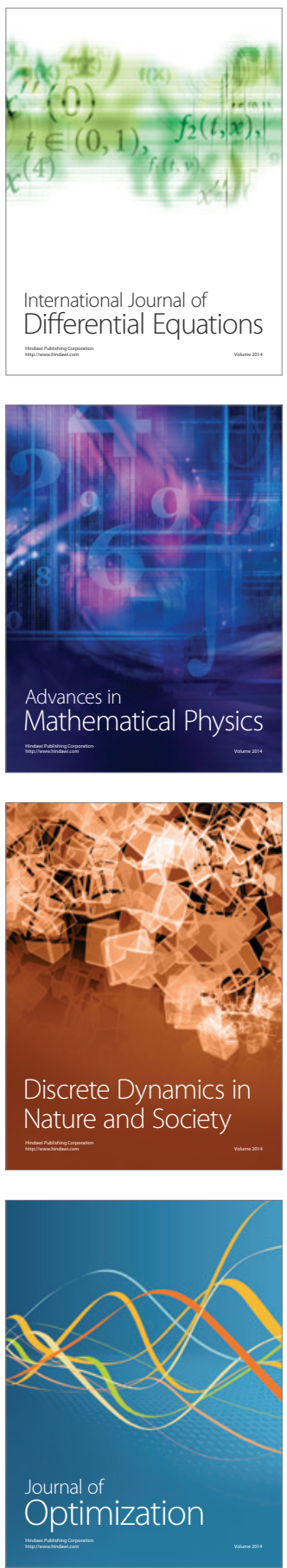